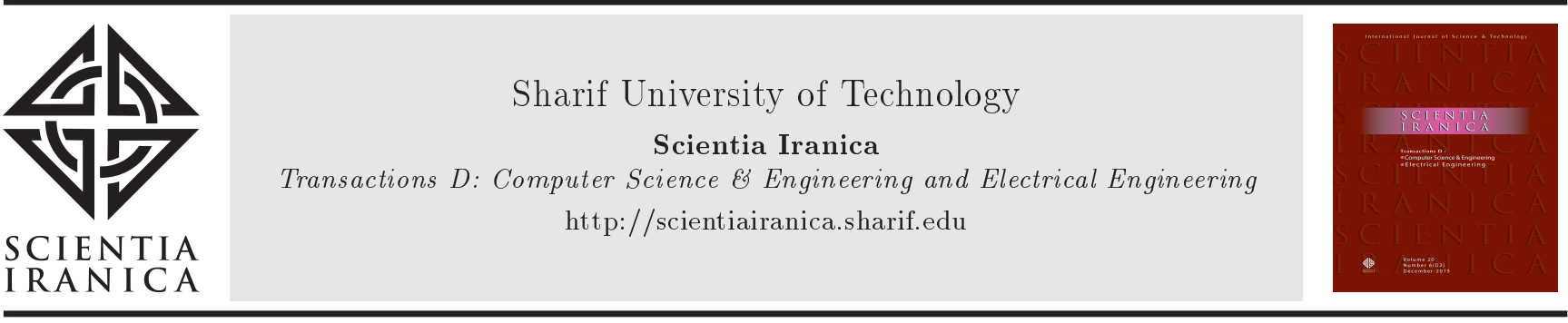

\title{
The comparative analysis of AC-flux and DC-flux resolvers
}

\author{
M. Bahari and Z. Nasiri-Gheidari* \\ Department of Electrical Engineering, Sharif University of Technology, Tehran, Iran. \\ Received 19 February 2020; received in revised form 20 June 2020; accepted 3 August 2020
}

\author{
KEYWORDS \\ Electromagnetic \\ sensor; \\ Variable Reluctance \\ (VR) resolver; \\ Time-Stepping Finite \\ Element Method \\ (TSFEM); \\ Hall-effect sensor; \\ External magnetic \\ field.
}

\begin{abstract}
Resolver, as an electromagnetic sensor, is widely used in many industrial applications. It can detect the position of the rotary part of the electric machines precisely. In commercial resolvers, excitation winding is connected to the High-Frequency (HF) AC source. The amplitude-modulated voltages induced in signal windings need to be demodulated in order to calculate the envelope of the output signals and detect the position accordingly. On the other hand, in PM-resolver, signal windings are replaced by Halleffect sensors to measure the DC magnetic flux, which is produced by permanent magnets. In this study, the performance of both $\mathrm{AC}$ and $\mathrm{DC}$ flux resolvers is investigated under different circumstances. All the simulations are conducted through the Time-Stepping Finite Element Method (TSFEM).
\end{abstract}

(C) 2022 Sharif University of Technology. All rights reserved.

\section{Introduction}

Position sensors play a crucial role in closed-loop motion control systems, especially in inverter-driven electric machines [1]. There are different types of position sensors, one of which is a resolver. Resolver is categorized as an electromagnetic position sensor and widely used in different industrial applications [1-3]. The main competitors to resolvers are optical encoders. Since optical encoders contain some delicate fragile parts, they cannot tolerate mechanical stresses and cannot be utilized in high-temperature environments. In addition, the performance of optical encoders is highly sensitive to the contamination while resolvers have acceptable performance in those conditions due to their robust structure. The working principle of resolvers is similar to the two-phase Synchronous

\footnotetext{
*. Corresponding author. Tel.: +982166164389 E-mail addresses: bahari.muhammad.93@gmail.com ( $M$. Bahari); znasiri@sharif.edu (Z. Nasiri-Gheidari)
}

Generators (2-ph SGs) and the only difference is that the excitation winding of the resolver is supplied by an $\mathrm{AC}$ voltage/current source rather than the DC one [3]. The windings of the traditional brushless resolvers are located in the slots of both the rotary and stationary parts. The induced voltage in the secondary coil of a Rotary Transformer (RT) fed the excitation winding, which is located in the slotted core of the rotary part. Using RT is characterized by such advantages as providing a contactless excitation supply $[4,5]$. However, it causes phase shift error and increases the size and the price of the resolver, as well. The proposed structures, nevertheless, use a great volume of copper and have a complicated manufacturing process. Eventually, Variable Reluctance (VR) resolvers are developed with a winding-less solid ferromagnetic rotor [6-8]. All of the aforementioned resolvers supply excitation winding with AC voltage/current source [7] and as a result, the AC-excited coils of excitation winding produce $\mathrm{AC}$ magnetic field that flows into the core of the sensor and induces HF voltage in signal windings. In addition to the radial flux resolvers, axial flux resolvers [9] and linear resolvers [10-12] have been investigated. In [13], 
a new PM-resolver with Permanent Magnets (PMs) in its structure was investigated which demonstrated that the resolver could also work properly with $\mathrm{DC}$ magnetic flux rather than $\mathrm{AC}$ magnetic flux. In the aforementioned resolver, $\mathrm{PMs}$ produced DC magnetic flux and due to the position of the sinusoidal-form 5 -X shape rotor, the sinusoidal magnetic flux flows through the Hall-effect sensors, which are located in the teeth instead of signal windings to measure the amplitude of flux density. DC flux resolver enjoys outstanding advantages such as lower cost due to the replacement of signal windings with cheap Hall-effect sensors and easy measurement of the position. Although the performance of a resolver with both $\mathrm{AC}$ and DC fluxes is well proved, no previous research has covered the comparative analysis of those two types of flux in resolvers. As a result, in this study, the performance of the AC magnetic flux and DC magnetic flux resolver with different types of excitation is investigated first. Due to similar conditions, the excitation winding of the DC magnetic flux resolver is connected to the DC supply and its performance is compared with the commercial AC magnetic flux resolver. Second, the impact of different constant speeds and different constant accelerations on the output signals is studied. Eventually, the performance of both AC flux resolver and DC flux resolver in the presence of an external magnetic field is examined. All the simulations are done using the time stepping finite element method.

\section{Studied resolver}

The stator and the coils of the commercial VR resolver, excited with AC supply, are shown in Figure 1(a). The conventional stator of the resolver is characterized by 12 slots, 4 excitation coils, and 8 signal coils. The stator, excitation coils, and Hall-effect sensors of the VR resolver excited with DC supply are shown in Figure 1(b). The stator of the DC excited resolver has 12 slots, 4 excitation coils, and 8 Hall-effect sensors
Table 1. The geometrical dimensions of the resolvers and supply characteristics.

\begin{tabular}{ccc}
\hline Geometrical dimensions & Unit & Value \\
\hline Parameters & & \\
\hline Pole Pairs $\left(p_{w}\right)$ & - & 5 \\
Air-gap length $($ min $\sim$ max $)$ & $\mathrm{mm}$ & $0.5 \sim 2$ \\
Shaft diameter & $\mathrm{mm}$ & 8 \\
Number of teeth $\left(N_{t}\right)$ & - & 12 \\
Stator outer diameter $\left(D_{o}\right)$ & $\mathrm{mm}$ & 40 \\
Stator inner diameter $\left(D_{i}\right)$ & $\mathrm{mm}$ & 24 \\
Total height & $\mathrm{mm}$ & 10 \\
Excitation winding's coil number & - & 50 \\
Signal winding's coil number & - & 50 \\
\hline Supply characteristics & \\
\hline Excitation voltage & $\mathrm{V}$ & 5 \\
Excitation current & $\mathrm{mA}$ & 50 \\
Excitation frequency (for AC supply) & $\mathrm{kHz}$ & 3.2 \\
\hline
\end{tabular}

(UGN3503). The rotor of both resolvers is 5 -X in shape, as shown in Figure 1(c). The geometrical dimensions of both resolvers and their other characteristics are listed in Table 1 . It is worth mentioning that the output voltages of the Hall-effect sensor can be obtained as follows:

$$
V_{s}=V_{o f f s e t}+\Delta V,
$$

where $V_{\text {offset }}$ depends on the amplitude of the voltage supply of the Hall-effect sensor and $\Delta V$ depends on the flux vector of the Hall-effect sensor divided by the area section of the sensor $\left(\frac{\vec{\varphi}}{A}\right)$. The output voltages of $h_{s 1}$, $h_{s 2}, h_{s 3}$, and $h_{s 4}$ Hall-effect sensors are combined to form the output voltage of the sine signal, and output voltages of $h_{s 1}, h_{s 2}, h_{s 3}$, and $h_{s 4}$ Hall-effect sensors are combined to form the output voltage of the cosine signal as follows:

$$
\begin{aligned}
& V_{s}=V_{s 1}+V_{s 2}-V_{s 3}-V_{s 4}, \\
& V_{c}=V_{c 1}+V_{c 2}-V_{c 3}-V_{c 4} .
\end{aligned}
$$
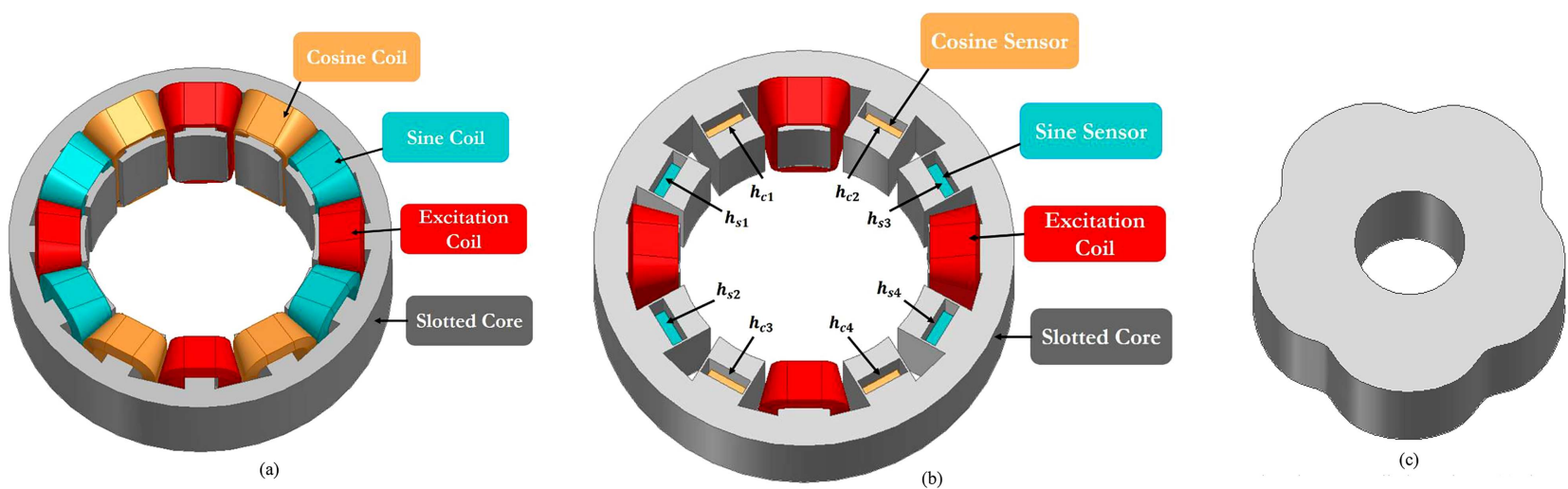

Figure 1. The conventional and DC supplied resolvers: (a) The stator of the conventional resolver, (b) the stator of the DC supplied resolver, and (c) the rotor of both resolvers. 
As can be seen in Eqs. (2) and (3), the offset voltages of the Hall-effect sensors are eliminated and the output voltages are only dependent on the magnitude of flux density of the sensors' section rather than the amplitude of Hall-effect sensor supply. As a result, even if the amplitude of the sensor supply varies, the sine signal and cosine signal as outputs will not be distorted, which proves the robustness of the resolver against amplitude changes of the sensors' supply.

\section{Different supply types}

Supplying the excitation winding with voltage or current source is a challenge for simulation of resolvers [10,14]. In this section, to investigate the performance of the resolvers, both $\mathrm{AC}$ flux resolver and DC flux resolver are simulated using TSFEM to study the influence of the excitation type on the output signals. Figure 2(a) shows the output signals of the conventional resolver with the $\mathrm{AC}$ voltage source excitation and Figure 2(b) shows the output signals of the aforementioned resolver with AC current source. As can be seen in Figure 2, the output signals of $\mathrm{AC}$ excited resolver are not affected by the type of voltage or current source. Figure 3(a) shows the output signals of the DC magnetic flux resolver excited with DC voltage source, and Figure 3(b) shows the output signals of the aforementioned resolver excited with DC current source. Despite the conventional resolver, the performance of the DC flux resolver is subjected to the supply type. As it can be seen in

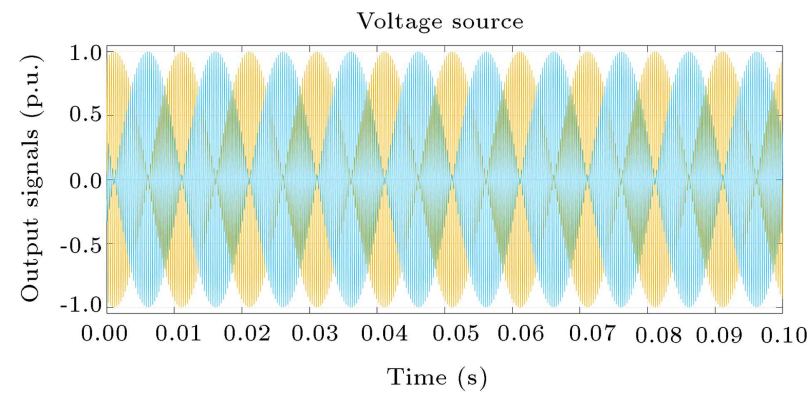

(a)

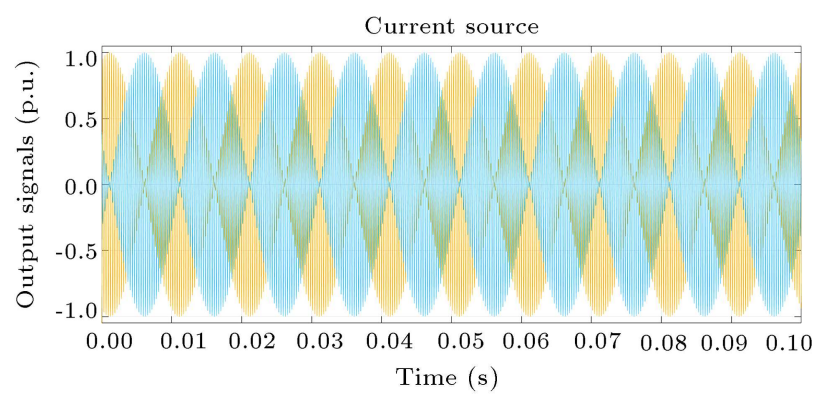

(b)

Figure 2. The commercial resolver with AC magnetic flux: (a) The output signals with voltage source excitation and (b) the output signals with current source excitation.

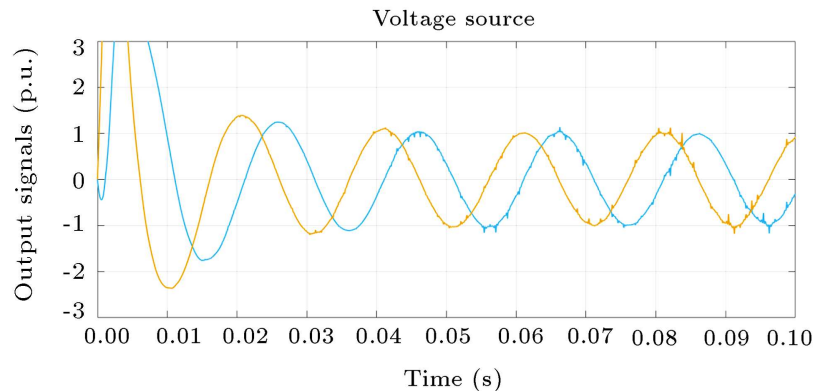

(a)

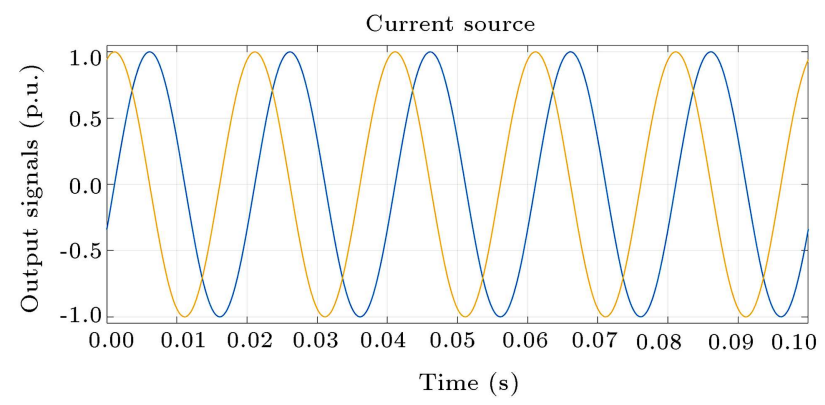

(b)

Figure 3. The DC magnetic flux resolver: (a) The output signals with voltage source excitation and (b) the output signals with current source excitation.

Figure 3(a), the output signals of voltage excitation suffer from slow dynamic, which means that when the mechanical speed of the rotary part of the resolver changes rapidly, it takes a long time for output signals (approximately $0.4 \mathrm{~ms}$ at $600 \mathrm{rpm}$ ) to get stable and it is disadvantageous to those applications that need a high-speed dynamic response. In addition, the accuracy of position detection is not sufficient and the Total Harmonic Distortion (THD) of the output signals is not acceptable. On the other hand, as it can be seen in Figure 3(b), the output signals of the DC flux resolver excited by current source have a desirable sinusoidal form and also get stable very fast. The Average of Absolute Position Error (AAPE) and Maximum Position Error (MPE) of both resolvers excited with voltage and current source are listed in Table 2 .

Table 2. The outputs of the AC and DC supplied resolvers with voltage/current source.

\begin{tabular}{cccc}
\hline $\begin{array}{c}\text { Excitation } \\
\text { type }\end{array}$ & $\begin{array}{c}\text { Supply } \\
\text { type }\end{array}$ & $\begin{array}{c}\text { AAPE } \\
\text { (deg.) }\end{array}$ & $\begin{array}{c}\text { MPE } \\
\text { (deg.) }\end{array}$ \\
\hline \multirow{2}{*}{ AC Excitation } & Voltage source & 0.1139 & 0.1978 \\
& Current source & 0.1265 & 0.3055 \\
& & & \\
DC excitation & Voltage source & 4.6935 & $>10$ \\
& Current source & 0.0316 & 0.0947 \\
\hline
\end{tabular}



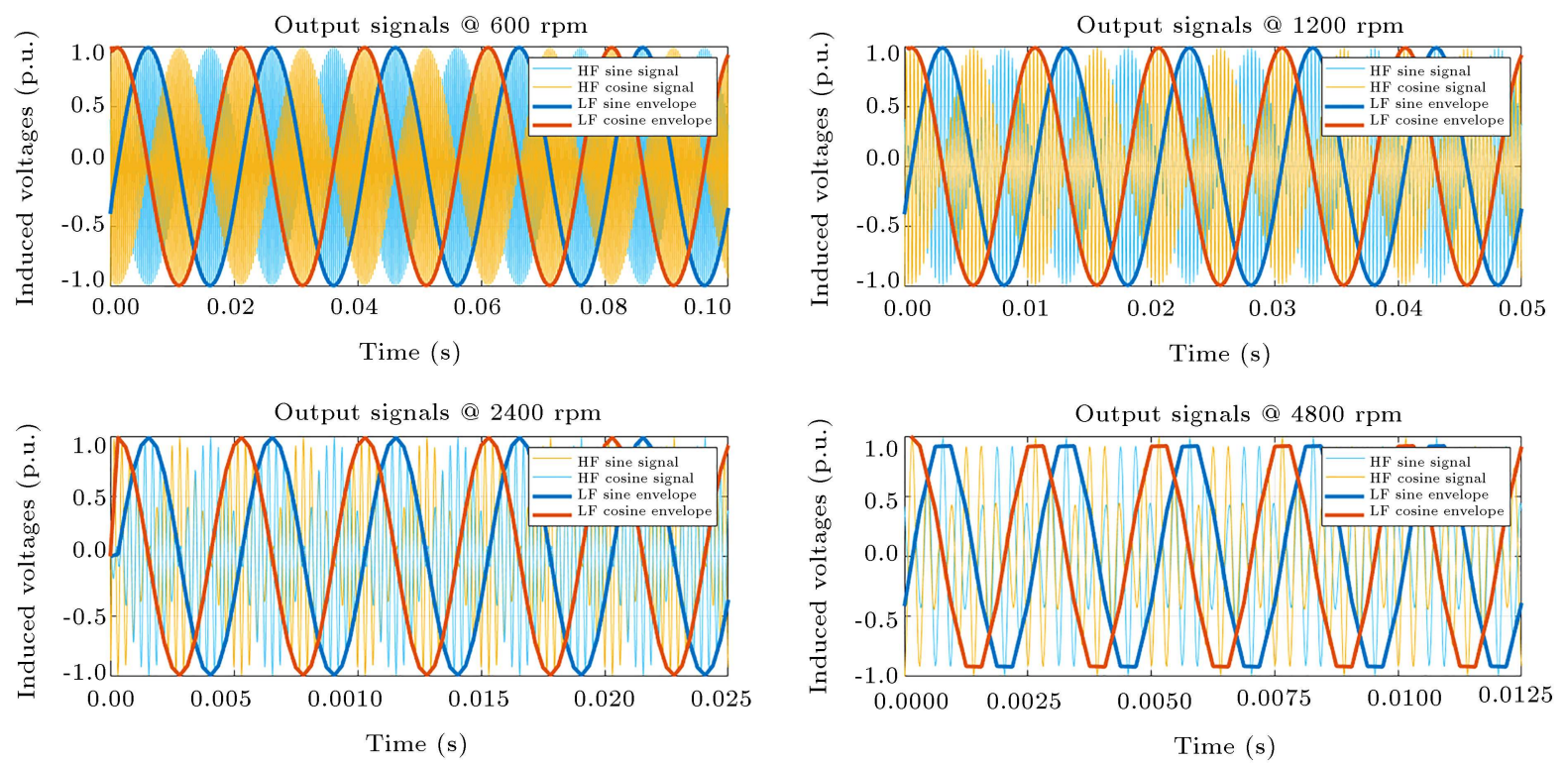

Figure 4. Induced voltages of AC flux resolver at different speeds.
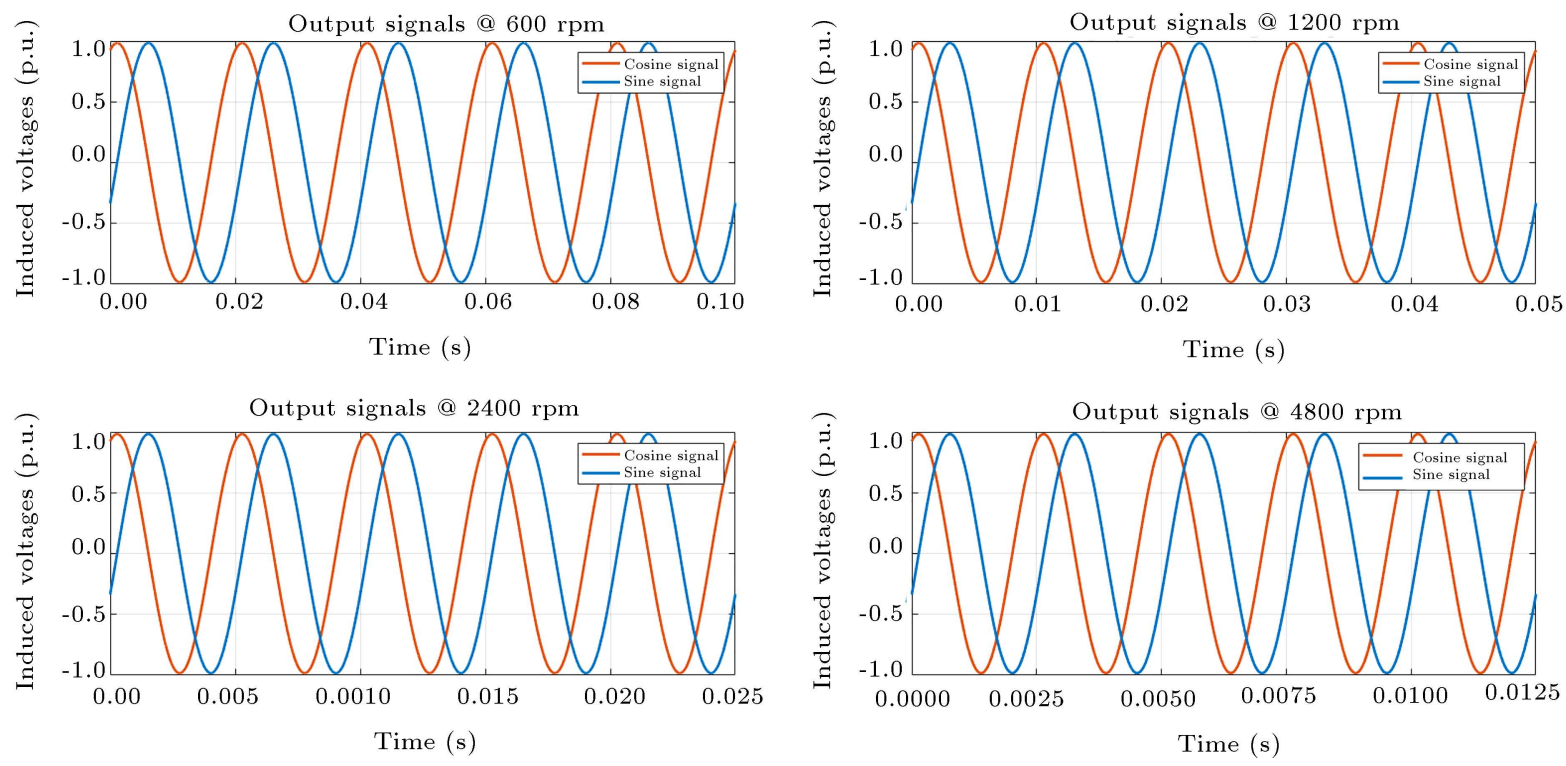

Figure 5. Output signals of DC flux resolver at different speeds.

\section{Impact of constant and variable speed}

Resolver as a position sensor should be able to work properly and calculate the angular position and angular speed of electric machines not only in a wide range of speeds but also in different accelerations. In this section, the performance of $\mathrm{AC}$ and $\mathrm{DC}$ resolvers is compared under constant/variable speed conditions. For the sake of fair comparison, the outputs of both resolvers with AC flux and DC flux are sampled with the same and constant sampling rates.

\section{a) Constant Speed}

For the AC flux resolver, the voltage source with the amplitude of $5 \mathrm{~V}$ and $3.2 \mathrm{kHz}$ supplies the excitation winding and for the DC flux resolver, the current source with an amplitude of $50 \mathrm{~mA}$ supplies the excitation winding. Simulations are repeated at $600,1200,2400$, and $4800 \mathrm{rpm}$. The results are presented in Figures 4 and 5 for $\mathrm{AC}$ and DC resolvers, respectively. The frequency of the sampling rate is $51.2 \mathrm{kHz}$ applied for both outputs. As can be seen in Figure 4, the quality of the Low Frequency (LF) envelope of the induced voltages in the $\mathrm{AC}$ flux resolver is reduced dramatically upon increase in the mechanical speed of the resolver. The main reason for this is that in each period of HF signal, only two extremum points of the LF envelope are sampled and the position of the rotary 
part is measured using the LF envelope in the peakdetection method.

$$
\begin{aligned}
& f_{H F \text { Signal-AC }}=n_{H F} \times f_{\text {exe }}, \quad n_{H F} \geq 16, \\
& f_{L F \text { Signal-AC }}=f_{\text {exe }} .
\end{aligned}
$$

In the DC flux resolver, nevertheless, all sampling points building the output signals of the resolver are used in calculating the position. As a result, the DC flux resolver kept the quality of its output signals, while the mechanical speed of the resolver increased.

$$
f_{L F \text { Signal-DC }}=f_{H F \text { Signal- }-A C} .
$$

Consequently, the quality of the output signals in the DC flux resolver is $\boldsymbol{n}_{\boldsymbol{H} \boldsymbol{F}}$ times better than that of the output signals in the AC flux resolver. The calculated AAPE and MPE at different speeds are listed in Table 3.

\section{b) Variable Speed}

To investigate the performance of both ACand DC-flux resolvers in variable speeds, AC flux resolver is simulated in two different angular accelerations. Figure 6(a) shows the real speed of the resolver as a reference and the calculated speed of the resolver using output signals of the AC flux resolver simultaneously when the speed of the rotary part increases with $628 \mathrm{rad} / \mathrm{s}^{2}$ and $1256 \mathrm{rad} / \mathrm{s}^{2}$. Figure $6(\mathrm{~b})$ shows the speed error of the aforementioned resolver. Simulations are repeated for the DC flux resolver with the same angular accelerations for the sake of fair comparison. Figure 7(a) shows the real speed and also the calculated speed of the DC flux resolver, and Figure 7(b) shows the speed error. Eventually, the Average of Absolute Speed Error (AASE) and Maximum Speed Error (MSE) of both resolvers with the same angular accelerations are listed in

Table 3. The outputs of the AC flux and DC flux resolvers at different constant speeds.

\begin{tabular}{ccccc}
\hline $\begin{array}{c}\text { Excitation } \\
\text { type }\end{array}$ & $\begin{array}{c}\text { Speed } \\
\text { (rpm) }\end{array}$ & $\begin{array}{c}\text { AAPE } \\
\text { (Deg.) }\end{array}$ & $\begin{array}{c}\text { MPE } \\
\text { (Deg.) }\end{array}$ & $\begin{array}{c}\text { THD } \\
(\mathbf{\%})\end{array}$ \\
\hline \multirow{4}{*}{ AC excitation } & 600 & 0.1139 & 0.1978 & 0.3338 \\
& 1200 & 0.1153 & 0.1983 & 0.3310 \\
& 2400 & 0.1247 & 0.2280 & 0.4625 \\
& 4800 & 0.2176 & 0.2823 & 0.7052 \\
DC excitation & 1200 & 0.0317 & 0.0998 & 0.0762 \\
& 2400 & 0.0317 & 0.1059 & 0.0766 \\
& 4800 & 0.0331 & 0.1113 & 0.0770 \\
\hline
\end{tabular}

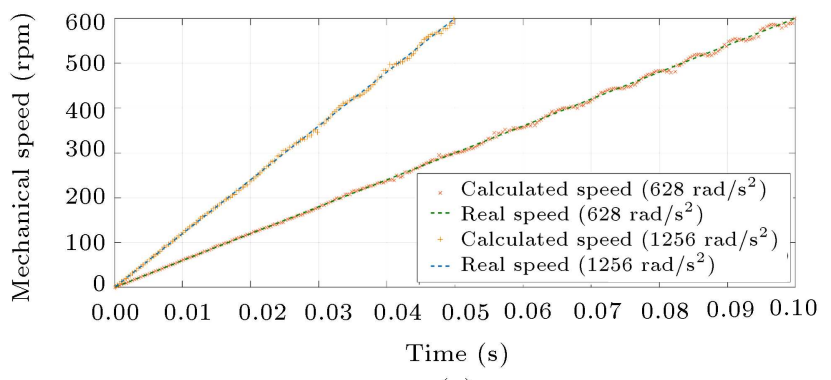

(a)

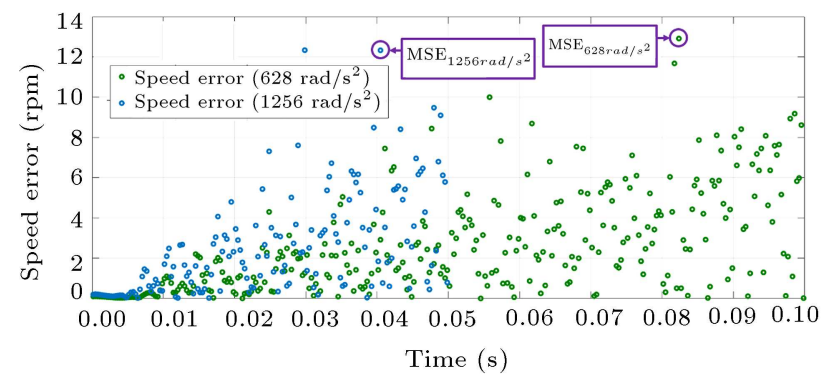

(b)

Figure 6. Performance of the AC flux resolver with two different angular accelerations: (a) Real speed of the resolver and calculated speed by output signals and (b) speed error.

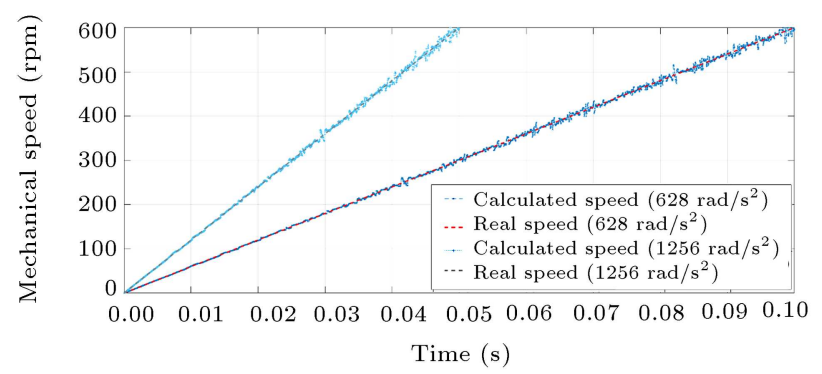

(a)

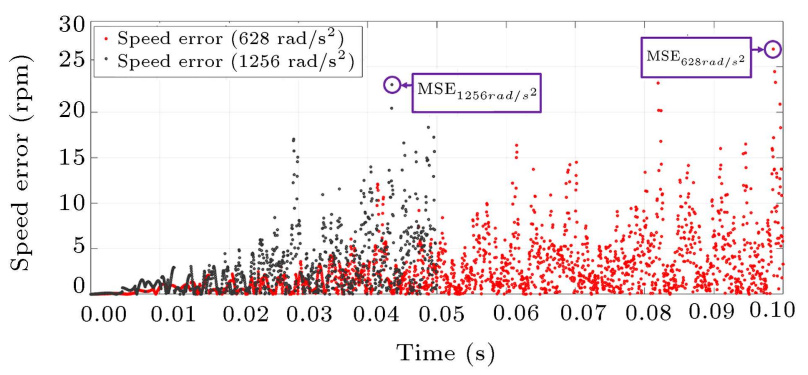

(b)

Figure 7. Performance of the DC flux resolver with two different angular accelerations: (a) Real speed of the resolver and calculated speed by output signals and (b) speed error.

Table 4. It can be concluded that both AC flux resolver and DC flux resolver operate well when the rotary part is accelerating. Although the AASE of both resolvers is almost equal, the MSE of the $\mathrm{DC}$ flux resolver is larger than the $\mathrm{AC}$ one. 
In conclusion, the $\mathrm{AC}$ flux resolver has a better performance than the DC flux resolver.

\section{Effect of external electromagnetic field on the performance of the resolver}

By distorting the magnetic flux that passes through the Hall-effect sensors, escalation of the resolver's position is inevitable. In order to study the influence of external electromagnetic interference on the performance of both resolvers, two intense asymmetric electromagnetic fields are simulated in the simulation environment: DC field with an amplitude of $2 \mathrm{k} \mathrm{A} / \mathrm{m}$, while another is the $\mathrm{AC}$ field of $16 \mathrm{kHz}$ with an amplitude of $2 \mathrm{k} \mathrm{A} / \mathrm{m}$. In these simulations, in order to provide similar conditions, both excitation windings of $\mathrm{AC}$ and DC flux resolvers are excited with the current source excitation of $50 \mathrm{~mA}$ amplitude and $3.2 \mathrm{kHz}$ frequency for the $\mathrm{AC}$ flux resolver. The outputs of the resolver including AAPE and MPE are listed in Table 5. As it can be seen, the position error of the $\mathrm{AC}$ flux resolver increases exponentially in the presence of the external electromagnetic field. Figure 8 shows distorted induced voltages in signal windings, especially in zero crossings. Due to the low amplitude of the signal in the zero-crossing section, the ratio of the signal-to-noise decreases and this leads to dramatic position error. Moreover, Figure 9 shows the output signals of the DC flux resolver in the presence of the external DC electromagnetic field. As can be seen, the external field distorts output signals of the DC flux

Table 4. The outputs of both AC and DC flux resolvers with different angular accelerations.

\begin{tabular}{cccc}
\hline $\begin{array}{c}\text { Excitation } \\
\text { type }\end{array}$ & $\begin{array}{c}\text { Angular } \\
\text { acceleration } \\
\left(\mathbf{r a d} / \mathbf{s}^{2}\right)\end{array}$ & $\begin{array}{c}\text { AASE } \\
(\mathbf{r p m})\end{array}$ & $\begin{array}{c}\text { MSE } \\
(\mathbf{r p m})\end{array}$ \\
\hline AC excitation & 628 & 2.56 & 12.91 \\
& 1256 & 2.54 & 12.33 \\
DC excitation & 628 & 2.61 & 26.92 \\
& 1256 & 2.62 & 23.01 \\
\hline
\end{tabular}

Table 5. The performance of the resolvers in the presence of external electromagnetic fields.

\begin{tabular}{cccc}
\hline $\begin{array}{c}\text { External magnetic } \\
\text { field }\end{array}$ & $\begin{array}{c}\text { Resolver } \\
\text { type }\end{array}$ & $\begin{array}{c}\text { AAPE } \\
\text { (deg.) }\end{array}$ & $\begin{array}{c}\text { MPE } \\
\text { (deg.) }\end{array}$ \\
\hline AC field $(2 \mathrm{kA} / \mathrm{m}-16 \mathrm{kHz})$ & AC flux & 1.58 & 2.83 \\
& DC flux & 0.34 & 0.96 \\
& & & \\
DC excitation $(2 \mathrm{kA} / \mathrm{m})$ & AC flux & 0.13 & 0.32 \\
& DC flux & 0.56 & 0.98 \\
\hline
\end{tabular}

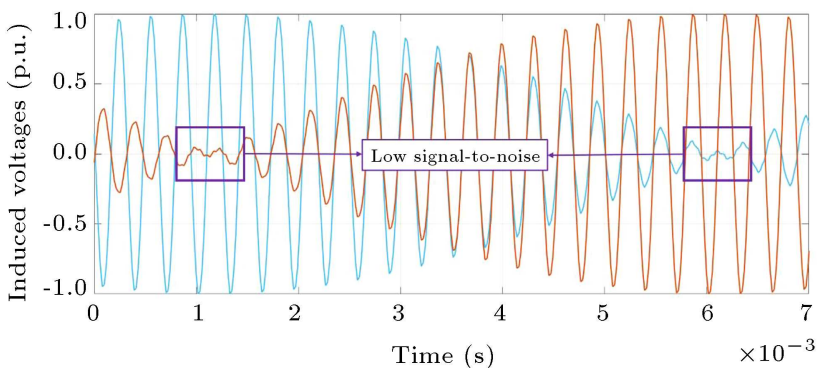

Figure 8. The effect of an AC external electromagnetic field on the induced voltages of the AC flux resolver.

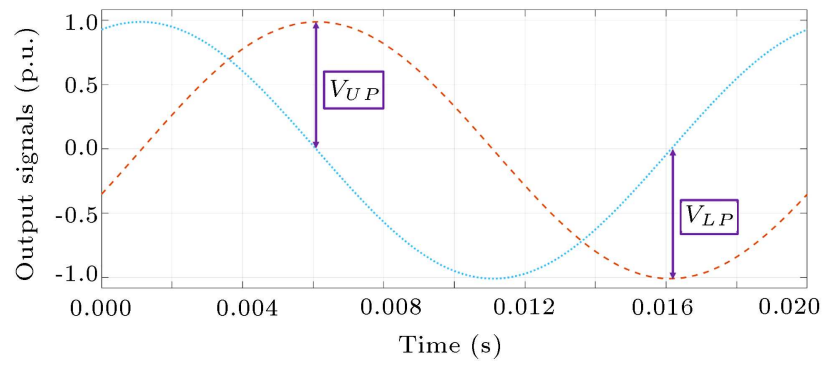

Figure 9. The effect of a DC external electromagnetic field on the DC flux resolver.

resolver by injecting offset to sine and cosine signal and consequently, causes increase in the position error of the resolver. The injected offset by the external field can be calculated as follows:

$$
\Delta V=V_{U P}-V_{L P}=0.022^{p . u .} .
$$

On the other hand, the DC electromagnetic field has minor effect on the performance of the $\mathrm{AC}$ flux resolver, and also the performance of the DC flux resolver in the presence of the $\mathrm{AC}$ electromagnetic field is acceptable.

\section{Conclusion}

In this paper, a comparative analysis of $\mathrm{AC}$ flux and DC flux resolvers was done. A variable reluctance resolver with a 12-slot stator and a 5-X-shaped rotor was taken as a study case. The position errors of both resolvers in normal conditions were examined when the current source and voltage source supplied the excitation winding and among different scenarios, DC flux resolver with the current source had the lowest AAPE and MPE. In order to study the accuracy in different conditions, both $\mathrm{AC}$ flux and DC flux resolvers were simulated in different ranges of speeds and accelerations. It was shown that the quality of output signals and their envelope in $\mathrm{AC}$ flux resolver reduced dramatically when the angular speed of the rotor increased. On the other hand, the DC flux resolver kept its acceptable accuracy even at high speeds. Although DC flux resolver 
proved its practicality in high-speed applications, the performance of both resolvers was close to each other in different angular accelerations. At last, the output signals of both resolvers were examined in the presence of external distortions. Simulation results indicated that AC flux resolvers almost kept their accuracy in case of an external DC magnetic field; however, an external AC magnetic field distorted their output signals. Moreover, DC flux resolver exhibits acceptable performance against an external AC magnetic field, while its accuracy is highly affected by an external DC magnetic field. To sum up, DC flux resolvers enjoy a great number of advantages including lower costs, easy position calculation, ability to measure the position at different speeds, and robust performance against $\mathrm{AC}$ distortions in comparison with commercial AC flux resolvers, which have complex winding configurations and as a result, deserve more attention.

\section{References}

1. Ge, X., Zhu, Z.Q., Ren, R., et al. "A novel variable reluctance resolver for $\mathrm{HEV} / \mathrm{EV}$ applications", IEEE Trans. Ind. Appl., 52(4), pp. 2872-2880 (2016).

2. Tootoonchian, F. "Design, performance, and testing of a brushless axial flux resolver without rotor windings", IEEE Sensors Journal, 16(20), pp. 7464-7471 (2016).

3. Murray, A., Hare, B., and Hirao, A. "Resolver position sensing system with integrated fault detection for automotive applications", Sensors, 2002 IEEE, Orlando, FL, USA, 2, pp. 864-869 (2002).

4. Sun, L. "Analysis and improvement on the structure of variable reluctance resolvers", IEEE Transactions on Magnetics, 44(8), pp. 2002-2008 (2008).

5. Mohammad-Yari, M., Safari, M., Alipour-Sarabi, R., et al. "Optimal winding selection for wound-rotor resolvers", Scientia Iranica, 28(6), pp. 3429-3436 (2021). DOI: $10.24200 /$ sci.2019.52439.2764

6. Ge, X. and Zhu, Z.Q. "A novel design of rotor contour for variable reluctance resolver by injecting auxiliary air-gap permeance harmonics", in IEEE Transactions on Energy Conversion, 31(1), pp. 345-353 (2016).

7. Sun, L. "Analysis and improvement on the structure of variable reluctance resolvers", in IEEE Transactions on Magnetics, 44(8), pp. 2002-2008 (2008).
8. Ge, X., Zhu, Z.Q., Ren, R., and Chen, J.T. "A novel variable reluctance resolver with nonoverlapping tooth-coil windings", IEEE Transactions on Energy Conversion, 30(2), pp. 784-794 (2015).

9. Alipour-Sarabi, R., Nasiri-Gheidari, Z., and Oraee, H. "Development of a 3-D magnetic equivalent circuit model for axial flux machines", IEEE Transactions on Industrial Electronics, 67(7), pp. 5758-5767 (2019).

10. Daniar, A., Nasiri-Gheidari, Z., and Tootoonchian, F. "Performance analysis of linear variable reluctance resolvers based on an improved winding function approach", IEEE Transactions on Energy Conversion, 33(3), pp. 1422-1430 (2018).

11. Bahari, M. and Nasiri-Gheidari, Z. "Longitudinal end effect in a variable area linear resolver and its compensating methods", Electrical Engineering (ICEE), Iranian Conference on, Mashhad, pp. 1316-1321 (2018).

12. Bahari, M., Alipour-Sarabi, R., Nasiri-Gheidari, Z., et al. "Proposal of winding function model for geometrical optimization of linear sinusoidal area resolvers", IEEE Sensors Journal, 19(14), pp. 5506-5513 (2019).

13. Bahari, M., Davoodi, A., Saneie, H., et al. "A new variable reluctance PM-resolver", IEEE Sensors Journal, 20(1), pp. 135-142 (2020).

14. Wang, K. and Wu, Z. "Hardware-based synchronous envelope detection strategy for resolver supplied with external excitation generator", IEEE Access, 7, pp. 20801-20810 (2019).

\section{Biographies}

Mohammad Bahari received the BSc degree from Semnan University, Semnan, Iran in 2015 and the MSc degree from the Sharif University of Technology, Tehran, Iran in 2019, all in Electrical Engineering. His research interests include design, modeling, and control of electrical machines, and finite-element analysis of electromagnetic devices.

Zahra Nasiri-Gheidari received the BSc degree from the Iran University of Sciences and Technology, Tehran, Iran in 2004 as well as the MS and PhD degrees from University of Tehran, Tehran in 2006 and 2012, respectively, all in Electrical Engineering. She is currently an Associate Professor at the Department of Electrical Engineering, Sharif University of Technology. Her research interests include design, optimization, and performance analysis of electrical machines and electromagnetic sensors. 